\title{
La grande transformation
}

\author{
Climat, inverserons-nous la courbe ?
}

Reinhold Leinfelder et un collectif

C et ouvrage est à mi-chemin entre un livre et une bande dessinée, en ce sens qu'il a une présentation de type BD, mais avec une densité de texte importante. Il met en scène une dizaine de personnages réels que les auteurs ont manifestement rencontrés et suivis pendant leurs cours, conférences ou visites de terrain. L'ouvrage rapporte donc leurs propos et les graphiques mettent les auteurs en scène, ou accompagnent le texte d'illustrations en rapport. Il en ressort un grand nombre de concepts et d'idées intéressants mais peu développés.

Le sujet porte sur le changement climatique et plus généralement l'environnement. Il commence par un constat que je trouve très pessimiste sur l'état de notre planète et présente ensuite un éventail de solutions qui me paraissent au contraire extrêmement optimistes. Les auteurs pensent manifestement qu'un développement technologique bien mené, accompagné de quelques évolutions sociétales, permettra un développement harmonieux. Ce développement va jusqu'à des tunnels transatlantiques sous vide permettant des déplacements à très grande vitesse grâce à la suspension magnétique, et ce dès 2050 , ce qui ne me paraît pas très crédible.

Les personnages mis en scène dans l'ouvrage, dont son éditeur, sont les membres du Conseil scientifique consultatif du gouvernement fédéral allemand sur les changements environnementaux mondiaux (WBGU). Une courte biographie de chacun d'entre eux est donnée en annexe. Ce sont tous des enseignants-chercheurs travaillant sur la thématique de l'environnement, mais avec des compétences très diverses, climatologue, ingénieur, économiste, politologue... Ils apportent donc des éclairages très divers sur cette thématique. Un accent particulier est mis sur le changement climatique, et donc la nécessité de diminuer notre consommation de combustibles fossiles et les émissions de gaz à effet de serre. Les auteurs semblent confiants que les besoins énergétiques de nos sociétés

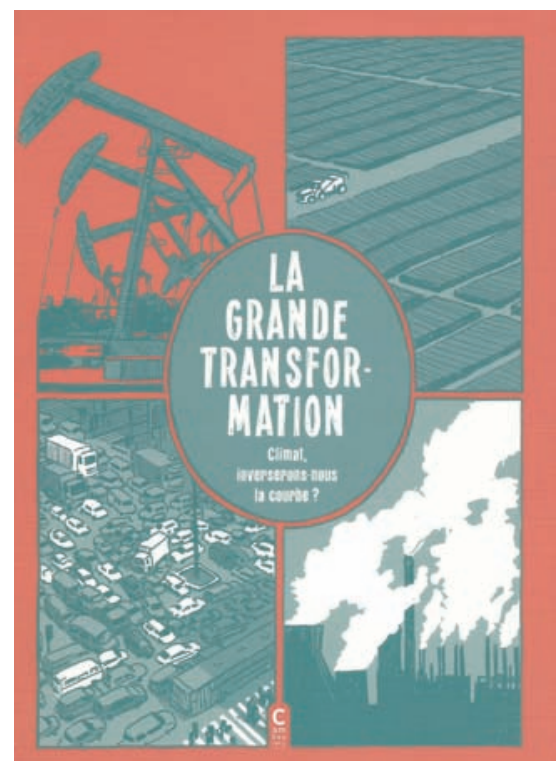

peuvent être couverts par des sources d'énergies renouvelables, éolien et solaire, le problème de l'intermittence étant couvert par la technique de méthanisation. Cette technologie, encore en développement, convertit l'électricité superflue en méthane, méthane qui est brûlé pour produire de l'électricité et de la chaleur lorsque nécessaire. Cette même technologie est envisagée par le rapport de l'Ademe qui envisage une électricité d'origine $100 \%$ renouvelable en France à l'échéance 2050. C'est une solution potentiellement prometteuse, bien que les rendements soient nécessairement limités, mais qui reste à démontrer à grande échelle.

L'intérêt de l'ouvrage est de discuter l'évolution nécessaire de nos sociétés pour lutter contre le changement climatique au-delà des aspects technologiques. Il lance ainsi quelques pistes sur la gouvernance, le financement, les évolutions sociétales qui devront se faire en parallèle aux développements technologiques. Il est ainsi mentionné la nécessité de manger moins de viande -rien d'original ici - et de privilégier la consommation d'insectes, ce qui paraît plus radical.

Les différents éclairages sur la question de la préservation de l'environnement sont indubitablement intéressants, mais la multiplicité implique qu'aucun n'est analysé dans le détail. L'ouvrage apporte donc plus des pistes de réflexion qu'une information complète. Il s'adresse à ceux qui s'intéressent au changement climatique et qui désirent avoir un regard original sur l'évolution souhaitable de nos sociétés occidentales. On peut ne pas être d'accord avec ces idées, mais elles méritent notre attention.

On notera dans cet ouvrage une préface d'Hervé Le Treut, directeur de l'IPSL, et une postface de Delphine Dissard, océanographe à l'IRD. Ces deux textes apportent eux aussi des pistes de réflexions intéressantes, et qui sont un peu plus développées (mais moins nombreuses) que celles de la section principale de l'ouvrage. De même, on trouve un glossaire assez complet qui va audelà d'une simple définition des termes en discutant quelques enjeux associés. La traduction depuis la version originale en allemand est excellente. J'ai juste noté l'usage surprenant du terme « huile minérale » là où « pétrole » serait attendu.

Ma principale critique sur cet ouvrage est qu'il aborde le problème du changement climatique presqu'uniquement sous l'angle des pays développés ; il est même très focalisé sur le cas de l'Allemagne. Le nucléaire n'est manifestement même pas envisagé comme pouvant apporter une énergie décarbonée dont notre société a besoin. Les quelques statistiques qui sont données sont spécifiques à l'Allemagne, alors que le changement climatique, et les actions pour le limiter, doivent se concevoir à une échelle mondiale.

\section{François-Marie Bréon}

La grande transformation. Climat,

inverserons nous la courbe?

par Reinhold Leinfelder et un collectif.

Traduit de l'allemand

Éditions Cambourakis, 2015, 144 p., $18 €$ 